Pacific

Journal of

Mathematics

RIGIDITY OF REPRESENTATIONS IN SO $(4,1)$ FOR DEHN FILLINGS ON 2-BRIDGE KNOTS

Stefano FrancaViglia AND JoAn Porti 


\title{
RIGIDITY OF REPRESENTATIONS IN SO $(4,1)$ FOR DEHN FILLINGS ON 2-BRIDGE KNOTS
}

\author{
SteFAnO Francaviglia AND JOAN PORTi
}

\begin{abstract}
We prove that, for a hyperbolic two-bridge knot, infinitely many Dehn fillings are rigid in $\mathrm{SO}_{0}(4,1)$. Here rigidity means that any discrete and faithful representation in $\mathrm{SO}_{0}(4,1)$ is conjugate to the holonomy representation in $\mathrm{SO}_{0}(3,1)$. We also show local rigidity for almost all Dehn fillings.
\end{abstract}

1. Introduction

2. Convergence of representations 252

3. Infinitesimal isometries and deformations 257

4. The variety of representations around $\rho_{0}$

5. Non-Fuchsian representations 267

Acknowledgments $\quad 273$

References $\quad 273$

\section{Introduction}

In this paper we consider compact, orientable three-manifolds $M$, whose boundary is a torus $\partial M \cong T^{2}$ and whose interior admits a complete hyperbolic metric of finite volume. We will specialize to the case where $M$ is the exterior of a hyperbolic twobridge knot and in particular its fundamental group is generated by two peripheral elements.

We tacitly assume that a basis for $H_{1}(\partial M ; \mathbf{Z}) \cong \mathbf{Z}^{2}$ has been fixed, and for $(p, q) \in \mathbf{Z}^{2}$ coprime, we denote by $M_{p / q}$ the manifold obtained by Dehn filling with meridian curve $(p, q)$. According to Thurston's hyperbolic Dehn filling theorem, for all but finitely many $p / q \in \mathbf{Q} \cup\{\infty\}$, the Dehn filled manifold $M_{p / q}$ is hyperbolic. In particular, its holonomy representation is the only discrete and faithful representation of $\pi_{1}\left(M_{p / q}\right)$ in $\mathrm{SO}_{0}(3,1)$ up to conjugation. Here $\mathrm{SO}_{0}(3,1)$ denotes the identity component of $\mathrm{SO}(3,1)$ and is isomorphic to $\operatorname{Isom}^{+}\left(\mathbf{H}^{3}\right)$, the orientation preserving isometry group of hyperbolic three space.

MSC2000: 57M50.

Keywords: fuchsian representation, global rigidity, two-bridge knot, cuspidal cohomology, Dehn

filling.

The first author was partially supported by the European Research Council (MERG-CT-2007046557) and the second one by grant FEDER/MEC MTM2006-04353. 
In this paper we address the question of whether $M_{p / q}$ has other discrete and faithful representations in $\mathrm{SO}_{0}(4,1) \cong \operatorname{Isom}^{+}\left(\mathbf{H}^{4}\right)$.

For the unfilled manifold $M$, M. Kapovich [1994] proved global rigidity. That is to say, every discrete and faithful representation of $\pi_{1}(M)$ in $\mathrm{SO}_{0}(4,1)$ is conjugated to a representation in $\mathrm{SO}_{0}(3,1)$, and therefore to the holonomy of the hyperbolic metric. Moreover Kapovich [1994] also proved infinitesimal rigidity for infinitely many Dehn fillings $M_{p / q}$, which are therefore locally rigid: there is no continuous nontrivial deformation in $\mathrm{SO}_{0}(4,1)$ of the holonomy of $M_{p / q}$. That result was then generalized by K. Scannell [2002] to Dehn fillings on a larger class of manifolds.

Here we prove global rigidity for infinitely many Dehn fillings on $M$, and local rigidity for almost all of them.

Theorem 1.1. Let $M$ be the exterior of a hyperbolic two-bridge knot. Then, for infinitely many $p / q \in \mathbf{Q} \cup\{\infty\}$, the Dehn filled manifold $M_{p / q}$ has no discrete and faithful representation in $\mathrm{SO}_{0}(4,1)$ other than its holonomy in $\mathrm{SO}_{0}(3,1)$.

The following definition can be found in [Scannell 2002]. Here we consider the action of the holonomy representation in the Lie algebra $\mathfrak{s o}(4,1)$ via the adjoint representation.

Definition 1.2. Let $M$ be a compact three manifold with boundary consisting of tori and whose interior is hyperbolic. Its parabolic cohomology is defined to be the kernel:

$$
\operatorname{PH}^{1}(M, \mathfrak{s o}(4,1))=\operatorname{ker}\left(H^{1}(M, \mathfrak{s o}(4,1)) \rightarrow H^{1}(\partial M, \mathfrak{s o}(4,1))\right) .
$$

Scannell [2002] proves that, for two-bridge knot exteriors,

$$
\operatorname{PH}^{1}(M, \mathfrak{s o}(4,1))=0 .
$$

The following improves a theorem of [Scannell 2002]:

Theorem 1.3. Let $M$ be a compact three manifold with boundary a torus whose interior is hyperbolic. If $\mathrm{PH}^{1}(M, \mathfrak{s o}(4,1))=0$, then almost all Dehn filled manifolds $M_{p / q}$ are locally rigid in $\mathrm{SO}(4,1)$.

Theorem 1.1 follows from Theorems 1.4 and 1.5 below. Theorem 1.3 is a consequence of the local analysis of the variety of representations made in the proof of Theorem 1.5.

Convergence of representations will be understood in the variety of representations of $M$, in particular algebraic convergence. For a discrete group $\Gamma$, the set of all representations of $\Gamma$ in $\mathrm{SO}_{0}(n, 1)$ is denoted by $R\left(\Gamma, \mathrm{SO}_{0}(n, 1)\right)$. It is well known that it is a real algebraic variety; see [Johnson and Millson 1987; Morgan 1986]. We are interested in representations up to conjugacy, but the space of conjugacy 
classes does not seem to have a structure easy to work with. For results about this set of conjugacy classes we refer to [Johnson and Millson 1987; Kapovich 1994; Morgan 1986; Scannell 2000].

When $\Gamma=\pi_{1}(M)$, it is customary to write

$$
R\left(M, \mathrm{SO}_{0}(n, 1)\right)=R\left(\pi_{1}(M), \mathrm{SO}_{0}(n, 1)\right) .
$$

Theorem 1.4. Let $M$ be a hyperbolic two-bridge knot exterior. Let $\rho_{0}: \pi_{1}(M) \rightarrow$ Isom $\left(\mathbf{H}^{4}\right)$ be the holonomy of the (Fuchsian) complete hyperbolic structure of $M$. Let $\left\{\rho_{n}\right\}_{n \in \mathbf{N}}$ be a sequence of representations

$$
\rho_{n}: \pi_{1}(M) \rightarrow \operatorname{Isom}\left(\mathbf{H}^{4}\right) .
$$

Suppose that, for each $n$, the representation $\rho_{n}$ factorizes through a discrete and faithful representation of $\pi_{1}\left(M_{p_{n} / q_{n}}\right)$ with $p_{n}^{2}+q_{n}^{2} \rightarrow \infty$. Then, up to conjugation, $\rho_{n}$ converges to $\rho_{0}$.

Theorem 1.5. Let $M$ be hyperbolic with one cusp with $\mathrm{PH}^{1}(M, \mathfrak{s o}(4,1))=0$. Let $\left\{\rho_{n}\right\}$ be a sequence of representations of $\pi_{1}(M)$ in $\mathrm{SO}_{0}(4,1)$ that converges to the holonomy of $M$.

If each $\rho_{n}$ comes from a discrete and faithful representation of $\pi_{1}\left(M_{p_{n} / q_{n}}\right)$, not conjugated to a representation in $\mathrm{SO}_{0}(3,1)$, then $p_{n} / q_{n} \rightarrow$ l for some $l \in \mathbf{R} \cup\{\infty\}$ depending only on $M$.

Here is the idea of the proof of Theorem 1.4. Take a sequence $\left\{\rho_{n}\right\}_{n \in \mathbf{N}}$, so that each $\rho_{n}$ is a discrete and faithful representation of $M_{p_{n} / q_{n}}$ with $p_{n}^{2}+q_{n}^{2} \rightarrow$ $\infty$. By theorems of [Bestvina 1988; Morgan and Shalen 1984; Paulin 1997], the space of discrete and faithful representations of $M$ in $\mathrm{SO}(4,1)$ is compact. Even if $\rho_{n}$ is not a faithful representation of $M$, the proof can be adapted to say that a subsequence of $\rho_{n}$ converges to $\rho$, a representation of $M$. Moreover, the socalled Chuckrow-Wielenberg's theorem can also be adapted to say that $\rho$ is discrete and faithful. Since $M$ is generated by two peripheral elements, by a theorem of M. Kapovich [1994] $\rho$ must be the holonomy representation of $M$ in $\operatorname{SO}(3,1)$.

Theorem 1.5 involves an analysis of $R(M, \mathrm{SO}(4,1))$ in a neighborhood of $\rho_{0}$, following closely the results obtained by Scannell [2002]. The tangent space to

$$
R\left(\pi_{1}(M), \mathrm{SO}_{0}(4,1)\right)
$$

is the space of cocycles valued on the Lie algebra $\mathfrak{s o}(4,1)$, and the space tangent to the orbits by conjugation is the space of coboundaries. Thus, to get relevant information, we study the cohomology group $H^{1}(M, \mathfrak{s o}(4,1))$. We need to understand how a sequence of representations in $\mathrm{SO}(4,1)$ can approach $\rho_{0}$, and we shall study which elements in $H^{1}(M, \mathfrak{s o}(4,1))$ are tangent vectors of deformations of 
$\rho_{0}$ in $R\left(\pi_{1}(M), \mathrm{SO}_{0}(4,1)\right)$, what in Section $4 \mathrm{~A}$ is called the differentiable tangent cone.

Since $\rho_{0}$ is contained in $\mathrm{SO}(3,1)$, the Lie algebra splits as $\pi_{1}(M)$-module as

$$
\mathfrak{s o}(4,1)=\mathfrak{s o}(3,1) \oplus \mathbf{R}^{3,1},
$$

where $\mathbf{R}^{3,1}$ denotes the Minkowski space equipped with $\operatorname{SO}(3,1)$ linear action. That splitting induces a direct sum of cohomology groups

$$
H^{1}(M, \mathfrak{s o}(4,1))=H^{1}(M, \mathfrak{s o}(3,1)) \oplus H^{1}\left(M, \mathbf{R}^{3,1}\right) .
$$

The subspace $H^{1}(M, \mathfrak{s o}(3,1))$ has dimension 2 and it is the tangent space to the variety of representations in $\mathrm{SO}(3,1)$ up to conjugation, described by Thurston's proof of the hyperbolic Dehn filling theorem; see [Kapovich 2001]. By a theorem of Scannell [2002], $H^{1}\left(M, \mathbf{R}^{3,1}\right)$ has dimension one. In Proposition 4.2, we show that the tangent cone is contained in the union

$$
H^{1}(M, \mathfrak{s o}(4,1))=H^{1}(M, \mathfrak{s o}(3,1)) \cup H^{1}\left(M, \mathbf{R}^{3,1}\right) .
$$

With the help of the curve selection lemma, this implies that if we have a sequence of non-Fuchsian representations approaching $\rho_{0}$, then the sequence must be contained in a semialgebraic set tangent to $H^{1}\left(M, \mathbf{R}^{3,1}\right)$.

In the proof we need to understand how elements in $H^{1}\left(\partial M, \mathbf{R}^{3,1}\right)$ are realized by deformations in the boundary $\partial M$, viewed as isometries of $\mathbf{R}^{3}=\partial \mathbf{H}^{4} \backslash\{\infty\}$. Those are realized by deforming a lattice in $\mathbf{R}^{2} \subset \mathbf{R}^{3}$ as a group of screw motions whose axis is contained in $\mathbf{R}^{2}$. This kind of deformation imposes some restriction on the Dehn filling coefficients, that we use to prove the theorem.

Our study of the variety of representations relies on previous work of Scannell [2002], where he shows that this is a singular point of the variety of representations.

The paper is organized in four sections. In Section 2 we prove Theorem 1.4. Section 3 is devoted to the preliminaries about infinitesimal deformations, and Section 4 to the analysis of a neighborhood of the variety of representations. The results of both sections are used in Section 5, where Theorem 1.5 is proved.

\section{Convergence of representations}

In this section we prove Theorem 1.4. Even if most of the arguments and techniques we use can be found in the literature, we give a proof for completeness, stressing the changes required in our situation. We mention in particular the recent work of M. Kapovich [2007] on convergence of groups.

Proof of Theorem 1.4. The proof goes through two main steps:

I The sequence $\left\{\rho_{n}\right\}$ is bounded in $R\left(\pi_{1}(M), \operatorname{Isom}\left(\mathbf{H}^{4}\right)\right)$; 
II each accumulation point of $\left\{\rho_{n}\right\}$ is discrete and faithful, whence conjugate to $\rho_{0}$ by [Kapovich 1994; Scannell 2002].

Each step is explained in a different subsection.

2A. Ultralimits and asymptotic cones. We concentrate here on Step I of the proof of Theorem 1.4.

We start by recalling the following result of Morgan and Shalen [1988].

Theorem 2.1. If $M$ is a complete hyperbolic 3-manifold, then there is no action of $\pi_{1}(M)$ on an $\mathbf{R}$-tree by isometries having

- no global fixed points, and

- small arc-stabilizers (that is, arc-stabilizers do not contain rank-two free subgroups).

We will show that, if $\left\{\rho_{n}\right\}$ was unbounded, then it would induce an action of $\pi_{1}(M)$ on an $\mathbf{R}$-tree as the one forbidden by Theorem 2.1, deducing therefore that $\left\{\rho_{n}\right\}$ must be bounded. The way to do that uses standard techniques of asymptotic cones (see for example [Kapovich 2001] for details on asymptotic cones).

Let $\omega$ be a nonprincipal ultrafilter, that we think of as a family of subsets of natural numbers such that:

(1) Given any subset $S \subset \mathbf{N}$, either $S \in \omega$ or $\mathbf{N} \backslash S \in \omega$;

(2) if $S \in \omega$, and $S^{\prime} \supset S$, then $S^{\prime} \in \omega$;

(3) if $S \subset \mathbf{N}$ is a finite subset, then $S \notin \omega$;

(4) if $S, S^{\prime} \in \omega$, then $S \cap S^{\prime} \in \omega$.

We say that a sequence $\left\{x_{n}\right\}$ in a topological space $\omega$-converges to a point $x$, and we write $\omega \lim x_{n}=x$, if for each open neighborhood $U$ of $x$, the set $\left\{i \in \mathbf{N}: x_{i} \in U\right\}$ belongs to $\omega$. It is an easy exercise that any sequence in a compact space has a unique $\omega$-limit.

Let $\left\{\gamma_{i}\right\}$ be a finite set of generators of $\pi_{1}(M)$. Let $*=\left\{*_{n}\right\}$ be a sequence of points such that $*_{n} \in \mathbf{H}^{4}$ realizes the minimum

$$
\min _{p \in \mathbf{H}^{4}} \max _{i} d\left(\rho_{n}\left(\gamma_{i}\right) p, p\right)
$$

and let

$$
\lambda_{n}=\max _{i} d\left(\rho_{n}\left(\gamma_{i}\right) *_{n}, *_{n}\right) .
$$

Let $(X, d)$ be the asymptotic cone of $\mathbf{H}^{4}$ made using the ultrafilter $\omega$, the sequence of rescaling parameters $\left\{\lambda_{n}\right\}$ and the base-points sequence $\left\{*_{n}\right\}$. Namely,

$$
X=\left\{\left\{x_{n}\right\} \subset \mathbf{H}^{4}: \omega \lim \frac{d\left(x_{n}, *_{n}\right)}{\lambda_{n}}<\infty\right\},
$$


where we identify two sequences $\left\{x_{n}\right\}$ and $\left\{y_{n}\right\}$ whenever

$$
\omega \lim \frac{d\left(x_{n}, y_{n}\right)}{\lambda_{n}}=0
$$

and we set:

$$
d\left(\left\{x_{n}\right\},\left\{y_{n}\right\}\right)=\omega \lim \frac{d\left(x_{n}, y_{n}\right)}{\lambda_{n}} .
$$

The following lemma is a standard fact about asymptotic cones of hyperbolic spaces; see for example [Kapovich 2001].

Lemma 2.2. If $\left\{\rho_{n}\right\}$ is unbounded, that is, if $\lambda_{n} \rightarrow \infty$, then $(X, d)$ is an $\mathbf{R}$-tree.

Lemma 2.3. The $\omega$-limit $\rho_{\omega}$ of $\left\{\rho_{n}\right\}$ is an isometric action of $\pi_{1}(M)$ on $X$, that is, a representation of $\pi_{1}(M)$ on $\operatorname{Isom}(X)$.

Proof. The action $\rho_{\omega}$ on $X$ is tautologically defined by

$$
\rho_{\omega}(\gamma)\left(\left\{x_{n}\right\}\right)=\left\{\rho_{n}(\gamma)\left(x_{n}\right)\right\} .
$$

We have to check that such a definition is well-posed; namely, that for all $\left\{x_{n}\right\} \in X$ and all $\gamma \in \pi_{1}(M)$ we have $\left\{\rho_{n}(\gamma)\left(x_{n}\right)\right\} \in X$. In other words, we need to check that

$$
\omega \lim \frac{d\left(x_{n}, *_{n}\right)}{\lambda_{n}}<\infty \quad \Longrightarrow \quad \omega \lim \frac{d\left(\rho_{n}(\gamma) x_{n}, *_{n}\right)}{\lambda_{n}}<\infty .
$$

Let $\gamma=\gamma_{i_{1}} \cdots \gamma_{i_{k}}$ be a decomposition of $\gamma$ in terms of the fixed generators of $\pi_{1}(M)$. Then

$$
\begin{aligned}
d\left(\rho_{n}(\gamma) x_{n}, *_{n}\right) & \leq d\left(\rho_{n}(\gamma) x_{n}, \rho_{n}(\gamma) *_{n}\right)+d\left(\rho_{n}(\gamma) *_{n}, *_{n}\right) \\
& =d\left(x_{n}, *_{n}\right)+d\left(\rho_{n}(\gamma) *_{n}, *_{n}\right) \\
& \leq d\left(x_{n}, *_{n}\right)+\sum_{j=1}^{k} d\left(\rho_{n}\left(\gamma_{i_{1}} \cdots \gamma_{i_{j}}\right) *_{n}, \rho_{n}\left(\gamma_{i_{1}} \cdots \gamma_{i_{j-1}}\right) *_{n}\right) \\
& \leq d\left(x_{n}, *_{n}\right)+k \max _{i} d\left(\rho_{n}\left(\gamma_{i}\right) *_{n}, *_{n}\right)=d\left(x_{n}, *_{n}\right)+k \lambda_{n} .
\end{aligned}
$$

Therefore $\rho_{\omega}$ is well defined. The fact that it is an action by isometries is obvious.

\section{Lemma 2.4. The representation}

$$
\rho_{\omega}: \pi_{1}(M) \rightarrow \operatorname{Isom}(X)
$$

has no global fixed point.

Proof. Let $\left\{x_{n}\right\}$ be any point of $X$. By construction of $*_{n}$ and $\lambda_{n}$, the index $i_{n}$ that realizes $\max _{i} d\left(\rho_{n}\left(\gamma_{i}\right)\left(x_{n}\right), x_{n}\right)$ satisfies

$$
d\left(\rho_{n}\left(\gamma_{i_{n}}\right)\left(x_{n}\right), x_{n}\right) \geq \lambda_{n} .
$$


Thus

$$
\frac{d\left(\rho_{n}\left(\gamma_{i_{n}}\right)\left(x_{n}\right), x_{n}\right)}{\lambda_{n}} \geq 1
$$

and therefore $d\left(\rho_{\omega}\left(\gamma_{\omega}\right)\left\{x_{n}\right\},\left\{x_{n}\right\}\right) \geq 1$, where $\gamma_{\omega}=\omega \lim \gamma_{i_{n}} \in\left\{\gamma_{i}\right\}$. It follows that $\left\{x_{n}\right\} \in X$ is not globally fixed.

Notice that the previous two lemmas, as well as the following one, do not use the hypothesis that $\left\{\rho_{n}\right\}$ is unbounded. Indeed, such hypothesis is only needed to show that $X$ is an $\mathbf{R}$-tree.

Lemma 2.5. The representation $\rho_{\omega}$ has small arc-stabilizers.

Proof. Let $I \subset X$ be an arc, and let $\Gamma<\pi_{1}(M)$ be its $\rho_{\omega}$-stabilizer:

$$
\rho_{\omega}(\Gamma)(I)=I .
$$

Let $\left\{x_{n}\right\}$ and $\left\{y_{n}\right\}$ be the end-points of $I$, and let $\gamma \in \Gamma$. Up to replacing $\gamma$ by $\gamma^{2}$ we have $\rho_{\omega}(\gamma)\left\{x_{n}\right\}=\left\{x_{n}\right\}$ and $\rho_{\omega}(\gamma)\left\{y_{n}\right\}=\left\{y_{n}\right\}$ as elements of $X$. That is to say,

$$
\omega \lim \frac{d\left(\rho_{n}(\gamma)\left(x_{n}\right), x_{n}\right)}{\lambda_{n}}=0
$$

and the same holds true for $y_{n}$. Since the $\omega$-limit of $d\left(x_{n}, y_{n}\right) / \lambda_{n}$ is the length of $I$, there exists a subsequence of indices $\left\{n_{k}\right\}$ such that

$$
\frac{d\left(\rho_{n_{k}}(\gamma)\left(x_{n_{k}}\right), x_{n_{k}}\right)+d\left(\rho_{n_{k}}(\gamma)\left(y_{n_{k}}\right), y_{n_{k}}\right)}{d\left(x_{n_{k}}, y_{n_{k}}\right)} \rightarrow 0 .
$$

Given any other $\psi \in \Gamma$, the same limit holds true up to subsequence. Now we use [Bestvina 1988, Proposition 4.5] (a Margulis-type argument, together with some hyperbolic trigonometry) to deduce that the group generated by $\rho_{n_{k}}(\gamma)$ and $\rho_{n_{k}}(\psi)$ is abelian for large enough $k$.

It follows that the commutator $[\gamma, \psi]$ belongs to the kernel of $\rho_{n_{k}}$ for large enough $k$.

Since $\rho_{n}$ factorizes through a faithful representation of $\pi_{1}\left(M_{p_{n} / q_{n}}\right)$, the following lemma shows that $[\gamma, \psi]$ is in fact trivial in $\Gamma$. This implies that $\Gamma$ is abelian, and therefore cannot contain rank-two free subgroups.

Lemma 2.6. Let

$$
P_{n}: \pi_{1}(M) \rightarrow \pi_{1}\left(M_{p_{n} / q_{n}}\right)
$$

denote the natural surjection induced by Dehn filling. If $p_{n}^{2}+q_{n}^{2} \rightarrow \infty$, then, for all $m \in \mathbf{N}$,

$$
\bigcap_{n>m} \operatorname{ker}\left(P_{n}\right)=1
$$


Proof. For large enough $n$, the Dehn filling on $M$ with parameters $\left(p_{n}, q_{n}\right)$ is hyperbolic by Thurston's hyperbolic Dehn filling theorem. Let

$$
\gamma \in \bigcap \operatorname{ker}\left(P_{n}\right)
$$

Then, the holonomy of $\gamma$ is trivial in each $M_{p_{n} / q_{n}}$. Again, by Thurston's theorem, the holonomy of $\gamma$ in $M_{p_{n} / q_{n}}$ converges to the holonomy of $\gamma$ in the complete hyperbolic structure of $M$, which is therefore trivial. This is possible only if $\gamma=1$.

So, $\rho_{\omega}$ is an isometric action on $X$ with no global fixed points and small arc stabilizers. By Theorem 2.1 such an action cannot exist. Therefore $X$ cannot be an $\mathbf{R}$-tree, whence we get that $\lambda_{n}$ must be bounded. Then, up to conjugation by isometries of $\mathbf{H}^{4}$, we can suppose that $*_{n}$ is constant, and the sequence $\left\{\rho_{n}\right\}$ is in that case bounded. This ends the proof of Step I.

2B. Accumulation point of Dehn fillings. We deal now with Step II of the proof of Theorem 1.4.

Let $\rho$ be an accumulation point of $\left\{\rho_{n}\right\}$. Let $V$ be an open neighborhood of the identity in $\operatorname{Isom}\left(\mathbf{H}^{4}\right)$ such that any discrete group finitely generated by elements in $V$ is virtually nilpotent. Such a $V$ exists by the Margulis lemma. Let $U$ be an open neighborhood of the identity such that $\bar{U} \subset V$.

Lemma 2.7. Let $\Gamma<\pi_{1}(M)$ be the subgroup generated by the elements $\gamma$ such that $\rho(\gamma) \in U$. Then $\Gamma$ is abelian.

Proof. Let $\Gamma_{0}<\Gamma$ be a group finitely generated by elements whose $\rho$-image is in $U$. For $n$ large enough, $\rho_{n}\left(\Gamma_{0}\right)$ is a discrete subgroup of $\operatorname{Isom}\left(\mathbf{H}^{4}\right)$, finitely generated by elements of $V$. Then, $\rho_{n}\left(\Gamma_{0}\right)$ is virtually nilpotent.

Since $\rho\left(\Gamma_{0}\right)$ is virtually nilpotent, it is also elementary, because the limit set of a nontrivial normal subgroup is the same as the limit set of the whole group. In particular, $\rho_{n}\left(\Gamma_{0}\right)$ is elementary. Moreover, $\rho_{n}\left(\Gamma_{0}\right)$ is torsion-free because $\rho_{n}$ is faithful as a representation of $\pi_{1}\left(M_{p_{n} / q_{n}}\right)$.

An elementary and torsion-free group of isometries in $\mathbf{H}^{4}$ must be either

- a subgroup of the stabilizer of a geodesic, $\mathbf{R} \rtimes O(3)$, or

- a parabolic subgroup fixing a point at $\partial \mathbf{H}^{4}$, that is, a subgroup of $\operatorname{Isom}\left(\mathbf{R}^{3}\right) \cong$ $\mathbf{R}^{3} \rtimes O(3)$.

In particular, all such groups are nilpotent of order two; see [Wolf 1984]. Thus, for $n$ large enough,

$$
\left[\left[\rho_{n}\left(\Gamma_{0}\right), \rho_{n}\left(\Gamma_{0}\right)\right], \rho_{n}\left(\Gamma_{0}\right)\right]=1 .
$$

It follows that any $\gamma \in \Gamma_{0}$ of the form $\gamma=\left[\left[\gamma_{1}, \gamma_{2}\right], \gamma_{3}\right]$ belongs eventually to $\operatorname{ker} \rho_{n}$, and by Lemma 2.6, this forces $\gamma$ to be trivial. That is to say, $\Gamma_{0}$ itself is 
virtually nilpotent. Since $\Gamma_{0}$ is a subgroup of the fundamental group of a hyperbolic manifold, this implies that $\Gamma_{0}$ is abelian. Since this holds for any $\Gamma_{0}$, we get that $\Gamma$ itself is abelian.

Corollary 2.8. The representation $\rho$ is faithful.

Proof. If $\operatorname{ker}(\rho)$ were not trivial, then it would be abelian by Lemma 2.7, but $\pi_{1}(M)$ has no abelian, nontrivial, normal subgroups.

The very same argument shows

Corollary 2.9. The representation $\rho$ is discrete.

Proof. Let $H_{0}$ be the connected component of the identity of the topological closure

$$
\overline{\rho\left(\pi_{1}(M)\right)} \text {. }
$$

As $\overline{\rho\left(\pi_{1}(M)\right)}$ is a Lie group, $H_{0}$ is normal in $\overline{\rho\left(\pi_{1}(M)\right)}$. It follows that

$$
\Gamma_{0}:=H_{0} \cap \rho\left(\pi_{1}(M)\right)
$$

is normal in $\rho\left(\pi_{1}(M)\right)$, and since $\rho$ is faithful, $\Gamma:=\rho^{-1}\left(\Gamma_{0}\right)$ is normal in $\pi_{1}(M)$.

On the other hand, $\Gamma_{0}$ is generated by elements in $U$, and then, by Lemma 2.7, $\Gamma$ is abelian. Therefore, $\Gamma=\{1\}$ and $H_{0}=\{1\}$, that is to say, $\rho$ is discrete.

This concludes Step II and so the proof of Theorem 1.4.

\section{Infinitesimal isometries and deformations}

This section contains the background material and tools that we need in the proof of Theorem 1.5.

Let $\mathbf{R}^{n, 1}$ denote the Minkowski space, that is, $\mathbf{R}^{n+1}$ equipped with the usual Lorentz product, that has matrix

$$
J=\left(\begin{array}{cccc}
-1 & 0 & & \\
0 & 1 & & \\
& & \ddots & \\
& & & 1
\end{array}\right) .
$$

We shall use the hyperboloid model for hyperbolic space

$$
\mathbf{H}^{n}=\left\{x=\left(\begin{array}{c}
x^{0} \\
\vdots \\
x^{n}
\end{array}\right) \in \mathbf{R}^{n, 1} \mid x^{t} J x=-1, x^{0}>0\right\}
$$

so that the orientation preserving isometry group of $\mathbf{H}^{n}$ is identified with the identity component of the group of linear transformations of $\mathbf{R}^{n+1}$ that preserve $J$ as a 
bilinear form:

$$
\operatorname{Isom}^{+}\left(\mathbf{H}^{n}\right)=\mathrm{SO}_{0}(n, 1) \text {. }
$$

We are interested in the cases $n=3$ and $n=4$. We shall consider the inclusion $\mathrm{SO}_{0}(3,1) \subset \mathrm{SO}_{0}(4,1)$ induced by the inclusion $\mathbf{R}^{3,1} \subset \mathbf{R}^{4,1}$ consisting in adding a fifth coordinate $x^{4}$. Namely,

$$
\begin{aligned}
\mathrm{SO}(3,1) & \rightarrow \mathrm{SO}(4,1), \\
A & \mapsto\left(\begin{array}{ll}
A & 0 \\
0 & 1
\end{array}\right) .
\end{aligned}
$$

3A. Infinitesimal isometries. The Lie algebra of $\mathrm{SO}(n, 1)$ is

$$
\mathfrak{s o}(n, 1)=\left\{a \in M_{n+1}(\mathbf{R}) \mid a^{t} J=-J a\right\} .
$$

Elements in $\mathfrak{s o}(n, 1)$ are viewed as infinitesimal isometries: a matrix $a \in \mathfrak{s o}(n, 1)$ is the tangent vector to the path $\exp (t a) \in \mathrm{SO}_{0}(n, 1)$ at $t=0$. The action of the isometry group on itself by conjugation induces the adjoint action of $\mathrm{SO}_{0}(n, 1)$ on the Lie algebra $\mathfrak{s o}(n, 1)$. Since we have an inclusion $\mathrm{SO}_{0}(3,1) \subset \mathrm{SO}_{0}(4,1)$, $\mathfrak{s o}(4,1)$ is also a $\mathrm{SO}_{0}(3,1)$-module.

Lemma 3.1. We have an isomorphism of $\mathrm{SO}_{0}(3,1)$-modules

$$
\mathfrak{s o}(4,1)=\mathfrak{s o}(3,1) \oplus \mathbf{R}^{3,1}
$$

where $\mathrm{SO}(3,1)$ acts on $\mathfrak{s o}(3,1)$ by the adjoint action and on $\mathbf{R}^{3,1}$ by the usual linear action.

Proof. Explicit construction. Given a matrix $a \in \mathfrak{s o}(3,1)$ and a (column) vector $v \in \mathbf{R}^{3,1}$, we consider the matrix

$$
\left(\begin{array}{cc}
a & v \\
-v^{t} J & 0
\end{array}\right) \in \mathfrak{s o}(4,1)
$$

where $v^{t}$ is the transpose vector. It is easy to check that this gives the isomorphism of the lemma, compatible with inclusion (3-1).

The Lie algebra of infinitesimal deformations can be identified with the Lie algebra of Killing vector fields.

Proposition 3.2. The subspace $\mathbf{R}^{3,1} \subset \mathfrak{s o}(4,1)$ corresponds to the Killing vector fields orthogonal to $\mathbf{H}^{3} \subset \mathbf{H}^{4}$.

Proof. Given $a \in \mathfrak{s o}(4,1)$, the corresponding field $V$ evaluated at $x \in \mathbf{H}^{4}$ is

$$
V_{x}=\left.\frac{d}{d t}\right|_{t=0} \exp (t a) x .
$$


Since we are working in a linear model, for $x \in \mathbf{H}^{4} \subset \mathbf{R}^{4,1}$,

$$
V_{x}=a x \in \mathbf{R}^{4,1} .
$$

In this model, $\mathbf{H}^{3}=\mathbf{H}^{4} \cap\left\{x^{4}=0\right\}$. Thus the Killing vector fields perpendicular to $\mathbf{H}^{3}$ correspond to matrices in $\mathfrak{s o}(4,1)$ whose entries are zero, except for the last column or the last row, which is the image of the embedding of $\mathbf{R}^{3,1}$ in $\mathfrak{s o}(4,1)$.

The splitting of Lemma 3.1 can be also understood by using the action on the de Sitter space

$$
\mathbf{S}^{3,1}=\left\{v \in \mathbf{R}^{4,1}: v^{t} J v=1\right\},
$$

which is naturally identified to the space of oriented hyperplanes in $\mathbf{H}^{4}$; see [Epstein and Penner 1988]. Since $\mathrm{SO}(4,1)$ acts transitively on $\mathbf{S}^{3,1}$ with stabilizers $\mathrm{SO}(3,1)$, we have that

$$
\mathrm{SO}(4,1) / \mathrm{SO}(3,1)=\mathbf{S}^{3,1} \text {. }
$$

Moreover the fibration

$$
\mathrm{SO}(3,1) \rightarrow \mathrm{SO}(4,1) \rightarrow \mathbf{S}^{3,1}
$$

whose projection maps $A \in \mathrm{SO}(4,1)$ to $A \cdot p$ for some fixed $p \in \mathbf{S}^{3,1}$, induces an exact sequence of $\mathfrak{s o}(3,1)$-modules:

$$
0 \rightarrow \mathfrak{s o}(3,1) \rightarrow \mathfrak{s o}(4,1) \rightarrow T_{p} \mathbf{S}^{3,1} \rightarrow 0
$$

which splits, by using either the Killing form or Lemma 3.1.

Remark 3.3. We have a canonical identification

$$
T_{p} \mathbf{S}^{3,1} \cong \mathbf{R}^{3,1}
$$

where $p \in \mathbf{S}^{3,1}$ is the hyperplane stabilized by $\mathfrak{s o}(3,1) \subset \mathfrak{s o}(4,1)$.

In order to be compatible with the previous computations, we shall assume that we choose the point

$$
p=\left(\begin{array}{l}
0 \\
0 \\
0 \\
0 \\
1
\end{array}\right) \in \mathbf{S}^{3,1}
$$


3B. The Zariski tangent space to the variety of representations. For a representation $\rho: \Gamma \rightarrow \mathrm{SO}_{0}(n, 1)$, the Zariski tangent space of the variety of representations at $\rho$ is naturally identified to the space of cocycles [Weil 1964]. Namely, the space of cocycles is defined as

$$
Z^{1}\left(\Gamma, \mathfrak{s o}(n, 1)_{\rho}\right)=\left\{d: \Gamma \rightarrow \mathfrak{s o}(n, 1) \mid d\left(\gamma_{1} \gamma_{2}\right)=d\left(\gamma_{1}\right)+\operatorname{Ad}_{\rho\left(\gamma_{1}\right)}\left(d\left(\gamma_{2}\right)\right)\right\} .
$$

Weil's correspondence maps the cocycle $d \in Z^{1}\left(\Gamma, \mathfrak{s o}(n, 1)_{\rho}\right)$ to the infinitesimal deformation

$$
\rho_{t}(\gamma)=(1+t d(\gamma)) \rho(\gamma),
$$

for all $\gamma \in \Gamma$, which is a representation modulo $t^{2}$, hence a Zariski tangent vector; see [Lubotzky and Magid 1985].

The space of coboundaries is

$$
\begin{aligned}
& B^{1}\left(\Gamma, \mathfrak{s o}(n, 1)_{\rho}\right)= \\
&\left\{d_{a}: \Gamma \rightarrow \mathfrak{s o}(n, 1) \mid d_{a}(\gamma)=a-\operatorname{Ad}_{\rho(\gamma)}(a), \text { for some } a \in \mathfrak{s o}(n, 1)\right\},
\end{aligned}
$$

and it is identified to the Zariski tangent space of orbits by conjugation.

The quotient is the cohomology group

$$
H^{1}\left(\Gamma, \mathfrak{s o}(n, 1)_{\rho}\right)=Z^{1}\left(\Gamma, \mathfrak{s o}(n, 1)_{\rho}\right) / B^{1}\left(\Gamma, \mathfrak{s o}(n, 1)_{\rho}\right) .
$$

Under some circumstances, $H^{1}\left(\Gamma, \mathfrak{s o}(n, 1)_{\rho}\right)$ can be viewed as the tangent space to the space of conjugacy classes of representations. However, for technical reasons, since $\mathbf{R}$ is not algebraically closed, it is easier to work with the variety of representations.

Now we focus on the case $\Gamma=\pi_{1}(M)$, where $M$ is a cusped manifold and $\rho_{0}$ is the holonomy representation of its complete structure. We omit the representation $\rho_{0}$ when writing the Lie algebra as $\pi_{1}(M)$-modules via the adjoint action of $\rho_{0}$. We also write $H^{1}(M, V)$ for $H^{1}\left(\pi_{1}(M), V\right)$. Recall that the parabolic cohomology is defined as

$$
\operatorname{PH}^{1}(M, \mathfrak{s o}(4,1))=\operatorname{ker}\left(H^{1}(M, \mathfrak{s o}(4,1)) \rightarrow H^{1}(\partial M, \mathfrak{s o}(4,1))\right) .
$$

Next lemma is due to Scannell [2002].

Lemma 3.4. Let $\rho_{0}: \pi_{1}(M) \rightarrow \mathrm{SO}_{0}(3,1) \subset \mathrm{SO}_{0}(4,1)$ be the holonomy representation of a hyperbolic two-bridge knot. Then

$$
\operatorname{PH}^{1}(M, \mathfrak{s o}(4,1))=0 .
$$

To prove that

$$
\operatorname{ker}\left(H^{1}(M, \mathfrak{s o}(4,1)) \rightarrow H^{1}(\partial M, \mathfrak{s o}(4,1))\right)
$$


vanishes, the idea is that an element in the kernel corresponds to a deformation that keeps the generators parabolic. By a geometric argument due to Kapovich and Scannell, such a representation must preserve a hyperbolic space of dimension three.

Lemma 3.5. Suppose that $\mathrm{PH}^{1}(M, \mathfrak{s o}(4,1))$ vanishes. Then the image of the inclusion

$$
0 \rightarrow H^{1}(M, \mathfrak{s o}(4,1)) \rightarrow H^{1}(\partial M, \mathfrak{s o}(4,1))
$$

has half dimension.

The proof consists in applying the long exact sequence of the pair and Poincaré duality.

Recall from Lemma 3.1 that, as $\rho_{0}$-module by the adjoint action, we have a decomposition

$$
\mathfrak{s o}(4,1)=\mathfrak{s o}(3,1) \oplus \mathbf{R}^{3,1},
$$

where $\mathbf{R}^{3,1}$ is the four-dimensional real vector space equipped with the linear action of $\operatorname{SO}(3,1) \subset G L(\mathbf{R}, 4)$. In particular,

$$
H^{1}(M, \mathfrak{s o}(4,1))=H^{1}(M, \mathfrak{s o}(3,1)) \oplus H^{1}\left(M, \mathbf{R}^{3,1}\right) .
$$

The dimensions of those spaces for the torus are well known.

Lemma 3.6 [Kapovich 1994; Scannell 2002].

$$
\operatorname{dim}\left(H^{1}(\partial M, \mathfrak{s o}(3,1))\right)=4, \quad \text { and } \quad \operatorname{dim}\left(H^{1}\left(\partial M, \mathbf{R}^{3,1}\right)\right)=2 .
$$

From Lemmas 3.6 and 3.5, we get:

Corollary 3.7. If $\mathrm{PH}^{1}(M, \mathfrak{s o}(4,1))=0$, then

$$
\operatorname{dim}\left(H^{1}(M, \mathfrak{s o}(3,1))\right)=2, \quad \text { and } \quad \operatorname{dim}\left(H^{1}\left(M, \mathbf{R}^{3,1}\right)\right)=1 .
$$

The following lemma can be easily proved using the formalism of the previous section. Recall that the projection $\mathrm{SO}(4,1) \rightarrow \mathbf{S}^{3,1}$ maps $A \in \mathrm{SO}(4,1)$ to $A \cdot p \in$ $\mathbf{S}^{3,1}$, for some fixed $p \in \mathbf{S}^{3,1}$.

Lemma 3.8. Let $\rho_{t}: \Gamma \rightarrow \mathrm{SO}(4,1)$ be a smooth path of representations of a group $\Gamma$ in $\mathrm{SO}(4,1)$, and let $\bar{\rho}_{t}$ be its projection to $\mathbf{S}^{3,1}$. Then, the first nontrivial derivative of $\bar{\rho}_{t}$ defines a cocycle in $Z^{1}\left(\Gamma, \mathbf{R}^{3,1}\right)$.

If the cocycle is nontrivial in $H^{1}\left(\Gamma, \mathbf{R}^{3,1}\right)$, then for small t the representation $\rho_{t}$ does not fix any hyperplane in $\mathbf{H}^{4}$.

Notice that this lemma uses the natural interpretation of points in $\mathbf{S}^{3,1}$ as hyperplanes in $\mathbf{H}^{4}$. 
3C. Trace functions. For $\gamma \in \Gamma$, let $\operatorname{tr}_{\gamma}: R\left(\Gamma, \mathrm{SO}_{0}(4,1)\right) \rightarrow \mathbf{R}$ denote the trace function. Since $\operatorname{tr}_{\gamma}$ is constant on orbits by conjugation, $d \operatorname{tr}_{\gamma}: Z^{1}(\Gamma, \mathfrak{s o}(4,1)) \rightarrow \mathbf{R}$ vanishes on $B^{1}(\Gamma, \mathfrak{s o}(4,1))$, and it induces a linear map, $d \operatorname{tr}_{\gamma}: H^{1}(\Gamma, \mathfrak{s o}(4,1)) \rightarrow$ R. (See also [Bart and Scannell 2007] for properties of the trace function.)

Lemma 3.9. For a representation $\rho: \Gamma \rightarrow \mathrm{SO}_{0}(3,1) \subset \mathrm{SO}_{0}(4,1)$, it holds

$$
H^{1}\left(\Gamma, \mathbf{R}^{3,1}\right) \subseteq \operatorname{ker} d \operatorname{tr}_{\gamma},
$$

for all $\gamma \in \Gamma$.

Proof. Using the embedding $\mathrm{SO}(3,1) \subset \mathrm{SO}(4,1)$ of (3-1), the corresponding embedding of $\mathbf{R}^{3,1}$ in $\mathfrak{s o}(4,1)$ maps the vector $v \in \mathbf{R}^{3,1}$ to the matrix

$$
\left(\begin{array}{cc}
0 & v \\
J v^{t} & 0
\end{array}\right)
$$

Then a path of representations $\rho_{t}$ tangent to a vector in $H^{1}\left(\Gamma, \mathbf{R}^{3,1}\right)$ can be written, up to fist order and up to conjugation, as

$$
\rho_{t}(\gamma)=\left(\operatorname{Id}+t\left(\begin{array}{cc}
0 & v \\
J v^{t} & 0
\end{array}\right)\right)\left(\begin{array}{cc}
\rho(\gamma) & 0 \\
0 & 1
\end{array}\right)+o\left(t^{2}\right)=\left(\begin{array}{cc}
\rho(\gamma) & t v \\
-t J v^{t} \rho(\gamma) & 1
\end{array}\right)+o\left(t^{2}\right),
$$

for all $\gamma \in \Gamma$. Hence $\operatorname{tr}\left(\rho_{t}(\gamma)\right)=\operatorname{tr}\left(\rho_{0}(\gamma)\right)+o\left(t^{2}\right)$ and $\left.\frac{d}{d t} \operatorname{tr}\left(\rho_{t}(\gamma)\right)\right|_{t=0}=0$.

Recall that $\operatorname{Isom}^{+}\left(\mathbf{H}^{3}\right) \cong P S L_{2}(\mathbf{C})$. This isomorphism comes from identifying the conformal sphere $S^{2}=\partial_{\infty} \mathbf{H}^{3}$ with the projective line $\mathbf{P}^{\mathbf{1}} \mathbf{C}$. The relation between traces in $\mathrm{SO}(3,1)$ and $S L_{2}(\mathbf{C})$ is:

Remark 3.10. Let $A \in \operatorname{Isom}^{+}\left(\mathbf{H}^{3}\right)$. Then,

$$
\operatorname{tr}_{\mathrm{SO}(3,1)}(A)=\left|\operatorname{tr}_{S L_{2}(\mathbf{C})}(A)\right|^{2} \quad \text { and } \quad \operatorname{tr}_{\mathrm{SO}(4,1)}(A)=\left|\operatorname{tr}_{S L_{2}(\mathbf{C})}(A)\right|^{2}+1 .
$$

Lemma 3.11. Let $\phi: \pi_{1}(M) \rightarrow P S L_{2}(\mathbf{C})$ be the holonomy representation of a hyperbolic manifold with one cusp. Then

(a) $H^{1}\left(M, \mathfrak{s l}_{2}(\mathbf{C})_{\mathrm{Ad} \phi}\right) \cong T_{\phi} X\left(M, P S L_{2}(\mathbf{C})\right) \cong \mathbf{C}$;

(b) for every $\gamma \in \pi_{1}(\partial M), \gamma \neq 1, d \operatorname{tr}_{\gamma}: H^{1}\left(M, \mathfrak{s l}_{2}(\mathbf{C})_{\mathrm{Ad} \phi}\right) \rightarrow \mathbf{C}$ is nonzero;

(c) given $\gamma_{1}, \gamma_{2} \in \pi_{1}(\partial M)$ so that

$$
\phi\left(\gamma_{i}\right)= \pm\left(\begin{array}{cc}
1 & x\left(\gamma_{i}\right) \\
0 & 1
\end{array}\right),
$$

then

$$
\frac{d \operatorname{tr}_{\gamma_{1}}}{d \operatorname{tr}_{\gamma_{2}}}=\left(\frac{x\left(\gamma_{1}\right)}{x\left(\gamma_{2}\right)}\right)^{2} .
$$


Proof. Assertions (a) and (b) are the contents of hyperbolic Dehn filling theorem; see [Kapovich 2001]. To prove (c), we follow [Boileau and Porti 2001, Appendix B] and, of course, Thurston's notes [1980]. We write a deformation as

$$
\phi_{t}\left(\gamma_{i}\right)= \pm\left(\begin{array}{cc}
e^{u_{i}(t)} & x\left(\gamma_{i}\right)(t) \\
0 & e^{-u_{i}(t)}
\end{array}\right) .
$$

The parameter $t$ is not a parameter of the deformation space because the trace function

$$
\operatorname{tr}_{\gamma_{i}}=e^{u_{i}(t)}+e^{-u_{i}(t)}=2 \cosh \left(u_{i}(t)\right)
$$

defines a local parameter of $R\left(M, S L_{2}(\mathbf{C})\right) / / S L_{2}(\mathbf{C})$. However one can take $t$ and $u_{i}(t)$ as analytic functions, by working in a double branched covering; see [Boileau and Porti 2001]. Following Thurston's notes, the commutativity relation becomes $x\left(\gamma_{1}\right) \sinh u_{2}=x\left(\gamma_{2}\right) \sinh u_{1}$. Thus

$$
\lim _{t \rightarrow 0} \frac{\dot{u}_{1}}{\dot{u}_{2}}=\lim _{t \rightarrow 0} \frac{u_{1}}{u_{2}}=\frac{x\left(\gamma_{1}\right)(0)}{x\left(\gamma_{2}\right)(0)},
$$

and (c) follows from straightforward computation.

\section{The variety of representations around $\rho_{0}$}

In this section we study the geometry of $R(M, \mathrm{SO}(4,1))$ in a neighborhood of the holonomy representation $\rho_{0}$ for the complete structure of $M$, namely the differentiable tangent cone in Section 4A, and the partial slice in Section 4B. Both tools are going to be used in the proof of Theorems 1.3 and 1.5.

4A. The differentiable tangent cone. There are several notions of tangent cone. For our purpose, we consider

Definition 4.1. The differentiable tangent cone of the variety of representations at $\rho_{0}$ is defined to be the subset of vectors in the Zariski tangent space $Z^{1}(M, \mathfrak{s o}(4,1))$ that are the tangent vectors to a curve of representations, parametrized by $[0, \varepsilon)$ and which is differentiable to the right at the origin.

As the cocycles project to cohomology, we look at the image of the differentiable tangent cone in

$$
H^{1}(M, \mathfrak{s o}(4,1)) \cong H^{1}(M, \mathfrak{s o}(3,1)) \oplus H^{1}\left(M, \mathbf{R}^{3,1}\right) .
$$

The aim of this section is to prove

Proposition 4.2. If $\mathrm{PH}^{1}(M, \mathfrak{s o}(4,1))=0$, then the image of the differentiable tangent cone in cohomology is contained in $H^{1}(M, \mathfrak{s o}(3,1)) \cup H^{1}\left(M, \mathbf{R}^{3,1}\right)$.

Before to prove it, we need a remark and a couple of lemmas. The remark can be proved by straightforward computation. 
Remark 4.3. Let $A \in \mathrm{SO}_{0}(4,1)$.

- If $A$ is parabolic and induces a translation in $\partial \mathbf{H}^{4} \backslash\{\infty\} \cong \mathbf{R}^{3}$, then

$$
\operatorname{trace}(A)=5 \text {. }
$$

- If $A$ is parabolic and induces a screw motion of angle $\alpha$ in $\partial \mathbf{H}^{4} \backslash\{\infty\}$, then

$$
\operatorname{trace}(A)=3+2 \cos \alpha .
$$

- If $A$ is elliptic with rotational angles $\alpha$ and $\beta$, then

$$
\operatorname{trace}(A)=1+2 \cos \alpha+2 \cos \beta .
$$

- If $A$ is loxodromic with translation length $\lambda$ and with angle $\alpha$, then

$$
\operatorname{trace}(A)=1+2 \cosh \lambda+2 \cos \alpha .
$$

In particular, if $A \in \mathrm{SO}_{0}(4,1)$ satisfies trace $(A)>5$, then $A$ is loxodromic.

The following lemma can be found in [Scannell 2002, Lemma 4.2].

Lemma 4.4. A representation of $\mathbf{Z} \times \mathbf{Z}$ in $\mathrm{SO}_{0}(4,1)$ containing loxodromic elements and obtained by perturbing a parabolic one (that consists only of translations in $\mathbf{R}^{3}$ ) must be conjugated to $\mathrm{SO}_{0}(3,1)$.

The next lemma is the analogue of Proposition 4.2 for $\pi_{1}(\partial M) \cong \mathbf{Z} \oplus \mathbf{Z}$, instead of $M$.

Lemma 4.5. Let $c:[0,1] \rightarrow R(\pi(\partial M), \mathrm{SO}(4,1))$ be a path differentiable to the right at 0 , and such that $c(0)$ is the restriction of the holonomy of the complete structure on $M$. Assume that the $\mathfrak{s o}(3,1)$-component of the projection of $\dot{c}(0)$ in $H^{1}(\partial M, \mathfrak{s o}(3,1)) \subset H^{1}(\partial M, \mathfrak{s o}(4,1))$ is not zero.

Then, there is $\varepsilon>0$ such that for all $0<t<\varepsilon, c(t)$ is conjugate to $\operatorname{SO}(3,1)$. In particular, the $\mathbf{R}^{3,1}$-component of $\dot{c}(0)$ vanishes.

Proof. Let us identify $\dot{c}(0)$ with its projection in cohomology. Let $u$ be the $\mathfrak{s o}(3,1)$ component of $\dot{c}(0)$, that is, $\dot{c}(0)-u \in H^{1}\left(\partial M, \mathbf{R}^{3,1}\right)$. By Lemma 3.9, for all $\gamma \in \pi_{1}(\partial M)$

$$
d \operatorname{tr}_{\gamma}(\dot{c}(0))=d \operatorname{tr}_{\gamma}(u) .
$$

As a representation in $\mathrm{PSL}_{2}(\mathbf{C}), c(0)$ is conjugated to

$$
\gamma \mapsto \pm\left(\begin{array}{cc}
1 & x(\gamma) \\
0 & 1
\end{array}\right)
$$

for all $\gamma \in \pi_{1}(\partial M)$. The map $x: \pi_{1}(\partial M) \rightarrow \mathbf{C}$ is a morphism and it defines a lattice of $\mathbf{C}$. From the formula

$$
\operatorname{tr}_{\mathrm{SO}(4,1)}=\operatorname{tr}_{\mathrm{SO}(3,1)}+1=\left|\operatorname{tr}_{S L(2, \mathbf{C})}\right|^{2}+1,
$$


and taking a lift such that $\operatorname{tr}_{S L(2, \mathbf{C}), \gamma}(c(0))=2$, we get

$$
d \operatorname{tr}_{\mathrm{SO}(4,1), \gamma}(u)=4 \operatorname{Re}\left(d \operatorname{tr}_{S L(2, \mathbf{C}), \gamma}(u)\right) .
$$

Since $x: \pi_{1}(\partial M) \rightarrow \mathbf{C}$ is a lattice, the set of arguments

$$
\left\{\frac{x(\gamma)}{|x(\gamma)|} \in S^{1}: \gamma \in \pi_{1}(\partial M) \backslash\{1\}\right\}
$$

is dense in the unit circle $S^{1}$. By Lemma 3.11 (c), and since $d \operatorname{tr}_{S L(2, \mathbf{C}), \gamma}(u) \neq 0$, one can find $\gamma$ such that $\operatorname{Re}\left(d \operatorname{tr}_{S L(2, \mathbf{C}), \gamma}(u)\right)>0$.

Therefore, for small enough $t$ the representation $c(t)$ contains loxodromic elements because of Remark 4.3, and is conjugated to $\mathrm{SO}(3,1)$ by Lemma 4.4.

Proof of Proposition 4.2. Since $\operatorname{PH}^{1}(M, \mathfrak{s o}(4,1))$ vanishes, the restriction from $\pi_{1}(M)$ to $\pi_{1}(\partial M)$ induces an inclusion in cohomology

$$
0 \rightarrow H^{1}(M, \mathfrak{s o}(4,1)) \rightarrow H^{1}(\partial M, \mathfrak{s o}(4,1)),
$$

and it is sufficient to show the corresponding statement for $\partial M$ instead of $M$. Namely, we have to check that "mixed" elements

$$
(u, v) \in H^{1}(\partial M, \mathfrak{s o}(3,1)) \oplus H^{1}\left(\partial M, \mathbf{R}^{3,1}\right)
$$

with $u \neq 0$ and $v \neq 0$ are not contained in the image of the differentiable tangent cone, and this is true by Lemma 4.5.

Remark 4.6. Further work would yield that the inverse image of the subspace $H^{1}\left(M, \mathbf{R}^{3,1}\right) \subset H^{1}(M, \mathfrak{s o}(4,1))$ in $Z^{1}(M, \mathfrak{s o}(4,1))$ is integrable, using the computations of Section 5C and an argument analogue to [Boileau et al. 2005, Theorem 9.4].

4B. The partial slice. Instead of working with the space of conjugacy classes of representations in $R(M, \mathrm{SO}(4,1))$, we shall construct a partial slice to the orbit by conjugation. Since $\mathrm{SO}(4,1)$ is a real group, the space of conjugacy classes is not an algebraic variety, though the partial slice is.

For $\rho \in R(M, \operatorname{SO}(4,1))$, the orbit of $\rho$ by conjugation is denoted by $O(\rho)$.

Proposition 4.7. There exists an algebraic subvariety $S \subset R(M, \mathrm{SO}(4,1))$ and an open neighborhood $U \subset R(M, \mathrm{SO}(4,1))$ of $\rho_{0}$, with the following properties:

(1) $S \cap O\left(\rho_{0}\right)=\left\{\rho_{0}\right\}$.

(2) If $\rho \in U$ satisfies that $\left.\rho\right|_{\partial M}$ fixes a point in $\partial \mathbf{H}^{4}$, then $O(\rho) \cap S \cap U \neq \varnothing$ and consists of a single point.

(3) The map $T S \rightarrow H^{1}(M, \mathfrak{s o}(4,1))$ is injective. 
(4) If $\rho \in U$ satisfies that $O(\rho) \cap R(M, \operatorname{SO}(3,1)) \neq \varnothing$, then

$$
O(\rho) \cap S \cap U \subset R(M, \operatorname{SO}(3,1)) .
$$

Proof. Let $\gamma_{1}, \gamma_{2}$ be a pair of peripheral elements that generate a nonelementary subgroup of $\pi_{1}(M)$. Define $S$ as the subset of representations $\rho$ in $R(M, \operatorname{SO}(4,1))$ that satisfy the conditions (a)-(d) below:

(a) $\rho\left(\gamma_{1}\right)$ fixes the same point of $\partial \mathbf{H}^{4}$ as $\rho_{0}\left(\gamma_{1}\right)$.

(b) $\rho\left(\gamma_{2}\right)$ fixes the same point of $\partial \mathbf{H}^{4}$ as $\rho_{0}\left(\gamma_{2}\right)$.

Let $\mu_{1} \in \pi_{1}(M)$ be an element such that $\gamma_{1}$ and $\mu_{1}$ generate a peripheral subgroup $\pi_{1}(\partial M)$. We fix an identification of $\partial \mathbf{H}^{4} \backslash \operatorname{Fix}\left(\rho_{0}\left(\gamma_{1}\right)\right)$ with $\mathbf{R}^{3}$, so that $\rho \in S$ restricted to $\mathbf{R}^{3}$ acts by affine transformations. Let $\overrightarrow{0} \in \mathbf{R}^{3}$ denote the origin, $\rho_{0}\left(\gamma_{1}\right)(\overrightarrow{0}) \neq \overrightarrow{0}$. The remaining conditions defining $S$ are:

(c) $\rho\left(\gamma_{1}\right)(\overrightarrow{0})=\rho_{0}\left(\gamma_{1}\right)(\overrightarrow{0})$.

(d) The ordered pairs of vectors

$$
\left(\rho\left(\gamma_{1}\right)(\overrightarrow{0}), \rho\left(\mu_{1}\right)(\overrightarrow{0})\right), \quad \text { and } \quad\left(\rho_{0}\left(\gamma_{1}\right)(\overrightarrow{0}), \rho_{0}\left(\mu_{1}\right)(\overrightarrow{0})\right)
$$

span the same oriented plane of $\mathbf{R}^{3}$.

Given a representation such that its restriction to $\partial M$ fixes a point in $\partial \mathbf{H}^{4}$, conditions (a) to (d) can be achieved by conjugation. Notice also that this determines the representation up to conjugacy. Namely, conditions (a) and (b) fix a representation of the conjugacy class, up to isometries that preserve a pair of points in $\partial \mathbf{H}^{4}=\mathbf{R}^{3} \cup\{\infty\}$. Hence we may assume that the fixed points are $\{\infty\}$ for $\rho\left(\gamma_{1}\right)$ in (a) and $\overrightarrow{0}$ for $\rho\left(\gamma_{2}\right)$ in (b). Thus the group of elements that fix those points is the product of the orthogonal group with the group of homotethies in $\mathbf{R}^{3}$, but this indeterminacy is eliminated by (c) and (d). Hence assertions (1) and (2) of the proposition follow.

The restrictions (a)-(d) can be written as $F^{-1}(0)$ for some map $F: U \rightarrow \mathbf{R}^{10}$ transverse to the orbit $O\left(\rho_{0}\right)$; hence $O\left(\rho_{0}\right)$ and $S$ are transverse.

$$
B^{1}(M, \mathfrak{s o}(4,1)) \cap T_{\rho_{0}} S=0,
$$

since $B^{1}(M, \mathfrak{s o}(4,1))$ is the tangent space to $O\left(\rho_{0}\right)$, and assertion (3) follows.

Finally, assertion (4) holds because uniqueness of assertion (2), and the fact that properties (a)-(d) may be achieved by conjugation in $\mathrm{SO}(3,1)$.

Notice that representations such that its restriction to $\partial M$ is contained in $\mathrm{SO}(4)$ are excluded by this set $S$, this is why we call it partial slice. 


\section{Non-Fuchsian representations}

This last section is devoted to the proof of Theorem 1.5. In Section 5A we deform a parabolic group of translations in the plane as a group of screw motions in Euclidean space. Viewing $\mathbf{R}^{3}$ as $\partial \mathbf{H}^{4} \backslash\{\infty\}$, those give infinitesimal deformations in $\mathfrak{s o}(4,1)$ that take values in $\mathbf{R}^{3,1}$. We claim in Lemma 5.4 that those are all possible deformations of $\mathbf{Z} \oplus \mathbf{Z}$ that take values in $\mathbf{R}^{3,1}$. Section $5 \mathrm{~B}$ is devoted to the proof of Theorem 1.5, assuming Lemma 5.4 which is proved in Section 5C.

5A. Deformations with peripheral screw motions. In this subsection we construct explicit examples of deformations of a parabolic representation of $\mathbf{Z} \oplus \mathbf{Z}$ that give cocycles valued in $\mathbf{R}^{3,1}$. The aim will be to show later that those are all the possible infinitesimal non-Fuchsian deformations.

Example 5.1 (Translation along a line as limits of rotations in the plane). Consider the translation that maps $x \in \mathbf{R}$ to $x+a$. We extend it to a translation of the plane with vector $(a, 0)^{t} \in \mathbf{R}^{2}$. For $a \in \mathbf{R}$, consider the family of rotations of $\mathbf{R}^{2}$ parametrized by $0<t<\varepsilon$, centered at the point $\left(0, \frac{1}{t}\right)^{t}$ and of angle $\alpha=a t$. They can be written as

$$
\left(\begin{array}{l}
x \\
y
\end{array}\right) \mapsto\left(\begin{array}{cc}
\cos (a t) & -\sin (a t) \\
\sin (a t) & \cos (a t)
\end{array}\right)\left(\begin{array}{l}
x \\
y
\end{array}\right)+\left(\begin{array}{c}
\sin (a t) / t \\
(1-\cos (a t)) / t
\end{array}\right),
$$

for all $(x, y)^{t} \in \mathbf{R}^{2}$. Obviously, when $t \rightarrow 0$, this converges to the translation of vector $(a, 0)^{t}$.

We now want to compute the derivative of this expression with respect to $t$. Consider a family of representations of $\mathbf{Z}$ that map 1 to the previous example. The corresponding cocycle maps 1 to the infinitesimal rotation

$$
\left(\begin{array}{cc}
0 & -a \\
a & 0
\end{array}\right)
$$

plus a vertical infinitesimal translation. The corresponding Killing vector field is perpendicular to the horizontal coordinate axis $\{(x, 0) \mid x \in \mathbf{R}\}$.

Example 5.2 (Translation along a plane as limits of rotations in the space). The same picture as above can be adapted for a group of translations of the plane by decomposing it as the orthogonal sum of two lines:

$$
\mathbf{R}^{2}=\langle\omega\rangle \oplus\langle\mathbf{i} \omega\rangle
$$

where $\omega \in \mathbf{R}^{2}$ satisfies $|\omega|=1, \mathbf{i} \omega$ denotes the result of rotating $\omega$ by $\pi / 2$, and identifying $\mathbf{R}^{2} \cong \mathbf{C}$. Then in the direction $\omega$ we do not make any deformation, and in the direction of $\mathbf{i} \omega$ we do the construction of Example 5.1. 
Assume we have a representation $\phi: \Gamma \rightarrow \mathbf{R}^{2}$ into the group of translations. Let rot $: \mathfrak{I s o m}\left(\mathbf{R}^{3}\right) \rightarrow \mathfrak{s o}(3)$ denote the projection induced by taking the linear

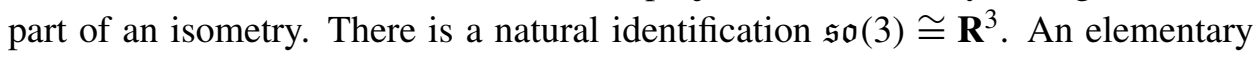
computation then shows:

Lemma 5.3. If $d$ is the cocycle of deformation in Example 5.2, then

$$
\operatorname{rot}(d(\gamma))=(\phi(\gamma) \cdot \mathbf{i} \omega) \omega
$$

for all $\gamma \in \Gamma$.

Here the dot denotes the Euclidean scalar product, so $\phi(\gamma) \cdot \mathbf{i} \omega$ denotes the orthogonal projection of $\phi(\gamma)$ in the direction perpendicular to $\omega$.

We are interested in the restriction of the holonomy $\rho_{0}$ of the complete structure, that we view as a representation into the group of translations $\rho_{0}: \pi_{1}(\partial M) \rightarrow \mathbf{R}^{2}$.

Lemma 5.4. (a) Any cocycle $d \in Z^{1}\left(\partial M, \mathbf{R}^{3,1}\right)$ takes values in $\mathbf{R}^{3,1} \cap \Im \mathfrak{s o m}\left(\mathbf{R}^{3}\right)$.

(b) Every cohomology class is represented by a cocycle d, so there exists a unit vector $\omega \in \mathbf{R}^{2}$, and a parameter $\lambda \in \mathbf{R}$ such that

$$
\operatorname{rot}(d(\gamma))=(\phi(\gamma) \cdot \mathbf{i} \omega) \lambda \omega,
$$

for all $\gamma \in \pi_{1}(\partial M)$. Moreover the cohomology class is trivial if and only if rot $\circ d$ is trivial.

The proof is postponed to Section 5C.

5B. Sequences of non-Fuchsian representations. For all $n \in \mathbf{N}$, let $\rho_{n}: \pi_{1}(M) \rightarrow$ $\mathrm{SO}(4,1)$ be a representation induced by a discrete and faithful representation of $M_{p_{n} / q_{n}}$ not conjugated to $\mathrm{SO}(3,1)$.

We claim that the restriction of $\rho_{n}$ to $\pi_{1}(\partial M)$ fixes a point in $\partial \mathbf{H}^{4}$, because its restriction to $\pi_{1}(\partial M)$ is a discrete and faithful representation of $\mathbf{Z}$, and therefore it cannot fix an interior point of $\mathbf{H}^{4}$. Thus we can apply Proposition 4.7, and assume, possibly up to conjugation, that $\rho_{n}$ belongs to the partial slice $S$.

From now on, all statements are up to subsequence. This can be done because the limit $l$ will depend only on $M$.

Lemma 5.5. There exists a cocycle $d \in T_{\rho_{0}} S$ and a sequence of positive real numbers $\varepsilon_{n} \rightarrow 0$ satisfying

$$
\rho_{n}(\gamma)=\left(1+\varepsilon_{n} d(\gamma)+o\left(\varepsilon_{n}\right)\right) \rho_{0}(\gamma)
$$

for all $\gamma \in \pi_{1} M$.

Here $o\left(\varepsilon_{n}\right)$ denotes a term such that $o\left(\varepsilon_{n}\right) / \varepsilon_{n} \rightarrow 0$. 
Proof. This is a compactness argument. Embed $R(M, \mathrm{SO}(4,1))$ in some $\mathbf{R}^{N}$ as an algebraic subvariety, let $\varepsilon_{n}$ be the distance between $\rho_{n}$ and $\rho_{0}$ and take a converging subsequence of unitary vectors $\frac{1}{\varepsilon_{n}}\left(\rho_{n}-\rho_{0}\right)$. The limit must be a vector Zariski tangent to $S$, and therefore it is a cocycle $d$.

Lemma 5.6. The cocycle d projects to a nontrivial element in $H^{1}(M, \mathfrak{s o}(4,1))$ contained in $H^{1}\left(M, \mathbf{R}^{3,1}\right)$.

Proof. By Proposition 4.7 (3) the cocycle $d$ is nontrivial in cohomology. Assume that it is not contained in $H^{1}\left(M, \mathbf{R}^{3,1}\right)$, we look for a contradiction by applying the curve selection lemma for semialgebraic sets.

Let $S \subset R(M, \mathrm{SO}(4,1))$ denote the slice of Proposition 4.7. Working with an embedding of $S \subset R(M, \operatorname{SO}(4,1))$ in the Euclidean space $\mathbf{R}^{N}$ and putting $\rho_{0}$ as the origin, let $\alpha>0$ denote the angle between $d$ and the linear subspace $Z^{1}\left(M, \mathbf{R}^{3,1}\right)$. Consider the semialgebraic cone $C$ consisting of those vectors of $\mathbf{R}^{N}$ whose angle with $Z^{1}\left(M, \mathbf{R}^{3,1}\right)$ is greater than or equal to $\alpha / 2$. By the curve selection lemma applied to $C \cap S \backslash R(M, \mathrm{SO}(3,1))$, there exists a semialgebraic curve $c:[0,1] \rightarrow S$ such that

(1) $c(0)=\rho_{0}$, and

(2) $c((0,1]) \subset C \cap S \backslash R(M, \mathrm{SO}(3,1))$.

The first nontrivial derivative $c^{(n)}(0)$ gives an element of the differential tangent cone whose projection to $H^{1}(M, \mathfrak{s o}(3,1))$ is nontrivial, by the choice of $\alpha$. Thus, by Proposition 4.2 applied to $c\left(t^{1 / n}\right)$, the cohomology class of $d$ must be contained in $H^{1}(M, \mathfrak{s o}(3,1))$.

Now, we argue with the inclusion of $\partial M$ in $M$ and the projection of $\operatorname{SO}(4,1)$ to the de Sitter space $\mathbf{S}^{3,1}=\mathrm{SO}(4,1) / \mathrm{SO}(3,1)$.

On one hand, by Lemma 4.5 the restriction of $c$ to $\partial M$ gives a path $c_{\partial}:[0,1] \rightarrow$ $R(\partial M, \mathrm{SO}(4,1))$ that must be contained in $\mathrm{SO}(3,1)$. In particular, the projection $\bar{c}_{\partial}$ of such path to $\mathbf{S}^{3,1}$ is the trivial path.

On the other hand, since $c$ is not contained in $R(M, \operatorname{SO}(3,1))$, its projection $\bar{c}$ to $\mathbf{S}^{3,1}$ must have some nontrivial derivative. This defines, by Lemma 3.8, a nontrivial cocycle $b \in Z^{1}\left(M, \mathbf{R}^{3,1}\right)$, which is nontrivial in $H^{1}\left(M, \mathbf{R}^{3,1}\right)$, because in the proof of Proposition 4.7, it is shown that $T_{\rho_{0}} S \cap B^{1}(M, \mathfrak{s o}(4,1))=0$, that is, the tangent space to the partial slice $S$ and the coboundary space are transverse. By Lemma 3.4, $b$ would give a nontrivial element when restricted to the boundary, contradicting the fact that $\bar{c}_{\partial}$ is the constant path.

Proof of Theorem 1.3. By contradiction, assume that there exists an infinite sequence $p_{n} / q_{n}$ so that $M_{p_{n} / q_{n}}$ is not locally rigid. Thus the holonomy of $M_{p_{n} / q_{n}}$ can be perturbed to $\tilde{\rho}_{n}: \pi_{1}\left(M_{p_{n} / q_{n}}\right) \rightarrow \mathrm{SO}(4,1)$, not contained in $\mathrm{SO}(3,1)$. Notice that since the holonomy of $M_{p_{n} / q_{n}}$ maps the core of the filling torus to a loxodromic 
element, we can assume that the restriction $\left.\tilde{\rho}_{n}\right|_{\partial M}$ is not elliptic. Thus $\tilde{\rho}_{n}$ belongs to the partial slice $S$ and the arguments of Lemmas 5.5 and 5.6 apply. In other words, there is a cocycle $d$ which is tangent to $\tilde{\rho}_{n}$, and that cocycle is contained in $H^{1}\left(M, \mathbf{R}^{3,1}\right)$.

However, since the $\tilde{\rho}_{n}$ are obtained by perturbation of the fuchsian holonomies of the manifolds $M_{p_{n} / q_{n}}$, and since the sequence of such holonomies defines a cocycle in $H^{1}(M, \mathfrak{s o}(3,1))$, we can choose the perturbations small enough so that the cocycle $d$ is not contained in $H^{1}\left(M, \mathbf{R}^{3,1}\right)$. Hence we get a contradiction.

Proof of Theorem 1.5. Let $\rho_{n}: \pi_{1}(M) \rightarrow \mathrm{SO}(4,1)$ be a sequence of representations induced by a discrete and faithful representation of $\pi_{1}\left(M_{p_{n} / q_{n}}\right)$, and let $d$ be as in Lemma 5.5. Since $d$ is tangent to $S$, we may assume that its restriction to $\pi_{1}(\partial M)$ takes values in the infinitesimal isometries of the Euclidean space $\mathbf{R}^{3}=\partial \mathbf{H}^{4} \backslash\left\{p_{0}\right\}$ by Lemma 5.4 (a). Thus, the composition with the projection $\mathfrak{I s o m}\left(\mathbf{R}^{3}\right) \rightarrow \mathfrak{s o}(3)$ gives a cocycle valued in infinitesimal rotations $\delta: \pi_{1}(\partial M) \rightarrow \mathfrak{s o}(3)$.

Since $d$ is not trivial in $H^{1}\left(\partial M, \mathbf{R}^{3,1}\right)$, by Lemma 5.4 (b), the image of $\delta$ is nontrivial and it has an invariant direction $\omega \in \mathbf{R}^{2} \subset \mathbf{R}^{3}$ with $|\omega|=1$.

The restriction of $\rho_{n}$ to $\partial M$ consists of screw motions of $\mathbf{R}^{3}$, and for each element $\gamma \in \pi_{1}(\partial M)$, the translation length of this screw motion is the product

$$
\operatorname{trans}\left(\rho_{n}(\gamma)\right)=\left(\rho_{n}(\gamma)(0)-(0)\right) \cdot \omega_{n},
$$

where $\omega_{n} \in \mathbf{R}^{3}$ is a unitary vector in the direction of the axis of the screw motion. Since the projection of $d$ is $\delta$, it follows that $\omega_{n} \rightarrow \omega$. Thus, given a system of peripheral generators $\left\langle\gamma_{1}, \gamma_{2}\right\rangle$, and the restriction

$$
p_{n} \operatorname{trans}\left(\rho_{n}\left(\gamma_{1}\right)\right)+q_{n} \operatorname{trans}\left(\rho_{n}\left(\gamma_{2}\right)\right)=0,
$$

we deduce the limit

$$
\lim _{n \rightarrow \infty} \frac{p_{n}}{q_{n}}=-\frac{\left(\rho_{0}\left(\gamma_{2}\right)(0)-(0)\right) \cdot \omega}{\left(\rho_{0}\left(\gamma_{1}\right)(0)-(0)\right) \cdot \omega}
$$

which is a well-defined element $l \in \mathbf{R} \cup \infty$, depending on the cusped manifold $M$ only.

Proof of Theorem 1.1. Let $C$ be the set of coefficients $(p, q)$ so that $\pi_{1}\left(M_{p / q}\right)$ has a discrete and faithful representation in $\mathrm{SO}(4,1)$, other than the holonomy of the complete hyperbolic structure of $M_{p / q}$. By Theorem 1.4, any sequence of pairwise distinct such representations must converge to the holonomy of the complete hyperbolic structure of $M$. By Theorem 1.5 it now follows that the set $C$ is asymptotic to the line $p / q=l$, where $l$ is a number - possibly $\infty$-depending only on $M$. Thus $C$ cannot be cofinite in the set of all filling coefficients. 
5C. Cohomology of $\mathrm{Z} \oplus \mathrm{Z}$ with coefficients in $\mathbf{R}^{\mathbf{3}, 1}$. The aim of this subsection is to prove Lemma 5.4 .

Before the proof we fix some notation. The restriction of the holonomy representation $\rho_{0}$ of $\pi_{1}(M)$ to $\pi_{1}(\partial M) \cong \mathbf{Z} \oplus \mathbf{Z}$ is a parabolic representation. Identifying the fixed point $p_{0}$ of $\left.\rho_{0}\right|_{\pi(\partial M)}$ with $\infty$, so that $\partial \mathbf{H}^{3}=\mathbf{R}^{2} \cup\left\{p_{0}\right\}$, the restriction is a representation by translations, that defines a lattice in the plane $\mathbf{R}^{2}$.

We choose

$$
p_{0}=\left(\begin{array}{l}
1 \\
1 \\
0 \\
0
\end{array}\right)
$$

to be the point of the light cone invariant by the holonomy of $\partial M$. With that choice, for $\gamma \in \pi_{1}(\partial M)$ if the translation vector of $\gamma$ is

$$
\operatorname{trans}\left(\rho_{0}(\gamma)\right)=(x, y) \in \mathbf{R}^{2},
$$

then the holonomy (as an element of $\mathrm{SO}(3,1)$ ) is

$$
\rho_{0}(\gamma)=\exp \left(\begin{array}{cccc}
0 & 0 & x & y \\
0 & 0 & x & y \\
x & -x & 0 & 0 \\
y & -y & 0 & 0
\end{array}\right)=\left(\begin{array}{cccc}
1+\frac{1}{2}\left(x^{2}+y^{2}\right) & -\frac{1}{2}\left(x^{2}+y^{2}\right) & x & y \\
\frac{1}{2}\left(x^{2}+y^{2}\right) & 1-\frac{1}{2}\left(x^{2}+y^{2}\right) & x & y \\
x & -x & 1 & 0 \\
y & -y & 0 & 1
\end{array}\right) .
$$

Elements in $\mathbf{R}^{3,1}$ may be written as

$$
v=\left(\begin{array}{c}
z+\lambda \\
z-\lambda \\
-\beta \\
\alpha
\end{array}\right)
$$

with $\lambda, z, \beta, \alpha \in \mathbf{R}$. Using the inclusion $\mathbf{R}^{3,1} \subset \mathfrak{s o}(4,1)$ of (3-2), the parameter $\lambda$ corresponds to the length of an infinitesimal displacement of $p_{0}$ in $\partial \mathbf{H}^{4}$ in the direction perpendicular to $\partial \mathbf{H}^{3}$.

In particular $\lambda=0$ defines the subspace of infinitesimal isometries that fix $p_{0}$, and therefore they restrict to infinitesimal similarities of $\mathbf{R}^{3}=\partial \mathbf{H}^{4} \backslash\left\{p_{0}\right\}$. Since our deformations vanish on the direction tangent to $\mathbf{R}^{2} \times\{0\}$ they must be infinitesimal isometries of Euclidean space. In fact we have:

$$
\mathbf{R}^{3,1} \cap \mathfrak{I s o m}\left(\mathbf{R}^{3}\right)=\left\{v \in \mathbf{R}^{3,1} \mid \lambda=0\right\} .
$$

The parameter $z$ describes the length of an infinitesimal translation in the direction perpendicular to $\mathbf{R}^{2}$. Finally, $\beta$ and $\alpha$ correspond to an infinitesimal rotation of 
vector $(\alpha, \beta, 0) \in \mathbf{R}^{2} \times\{0\}$. Using the coordinates in (5-1), the projection

$$
\operatorname{rot}: \mathfrak{I s o m}\left(\mathbf{R}^{3}\right) \rightarrow \mathfrak{s o}(3) \cong \mathbf{R}^{3}
$$

restricts to

$$
\begin{aligned}
\operatorname{rot}:\{\lambda=0\} \subset \mathbf{R}^{3,1} & \rightarrow \mathbf{R}^{2} \times\{0\}, \\
v & \mapsto(\alpha, \beta, 0) .
\end{aligned}
$$

Here rot denotes the tangent map of the epimorphism $\operatorname{Isom}\left(\mathbf{R}^{3}\right) \rightarrow O(3)$.

Proof of Lemma 5.4. Fix a system of generators $g_{1}, g_{2}$ for $\pi_{1}(\partial M)$, so that $\rho_{0}\left(g_{1}\right)$ is a translation of vector $\left(x_{1}, y_{1}\right) \in \mathbf{R}^{2}$, and $\rho_{0}\left(g_{2}\right)$, of vector $\left(x_{2}, y_{2}\right) \in \mathbf{R}^{2}$.

For a cocycle $d \in Z^{1}\left(\partial M ; \mathbf{R}^{3,1}\right)$, define $\lambda_{1}, \lambda_{2}, \alpha_{1}, \alpha_{2}, \beta_{1}, \beta_{2}, z_{1}$ and $z_{2} \in \mathbf{R}$ so that

$$
d\left(g_{i}\right)=\left(\begin{array}{c}
z_{i}+\lambda_{i} \\
z_{i}-\lambda_{i} \\
-\beta_{i} \\
\alpha_{i}
\end{array}\right),
$$

for $i=1,2$. By Fox calculus (see, for example, [Lubotzky and Magid 1985]) the parameters $\alpha_{i}, \beta_{i}, z_{i}$ and $\lambda_{i}$ are subject to the relation $\left(g_{1}-1\right) d\left(g_{2}\right)=\left(g_{2}-1\right) d\left(g_{1}\right)$, which is equivalent to

$$
\lambda_{1}=\lambda_{2}=0, \quad \text { and } \quad-\beta_{2} x_{1}+\alpha_{2} y_{1}=-\beta_{1} x_{2}+\alpha_{1} y_{2},
$$

that give a five-dimensional real space on the parameters subject to these relations. In particular since $\lambda_{1}=\lambda_{2}=0$, statement (a) is proved.

Notice that $d$ is a coboundary if and only if there exist parameters $A, B, L \in \mathbf{R}$ such that

$$
d\left(g_{i}\right)=\left(\begin{array}{c}
L\left(x_{i}^{2}+y_{i}^{2}\right)-B x_{i}+A y_{i} \\
L\left(x_{i}^{2}+y_{i}^{2}\right)-B x_{i}+A y_{i} \\
2 L x_{i} \\
2 L y_{i}
\end{array}\right),
$$

for $i=1,2$.

It follows immediately that if $\alpha_{1}=\alpha_{2}=\beta_{1}=\beta_{2}=0$, then $d$ is a coboundary.

Now the remaining of the proof is an elementary but tricky computation. The equality

$$
-\beta_{2} x_{1}+\alpha_{2} y_{1}=-\beta_{1} x_{2}+\alpha_{1} y_{2}
$$

may be seen as an equality of imaginary parts:

$$
\operatorname{Im}\left(\left(x_{1}-\mathbf{i} y_{1}\right)\left(\alpha_{2}+\mathbf{i} \beta_{2}\right)\right)=\operatorname{Im}\left(\left(x_{2}-\mathbf{i} y_{2}\right)\left(\alpha_{1}+\mathbf{i} \beta_{1}\right)\right) .
$$

Next we claim that, by adding a cocycle, we can remove imaginary parts. Namely the expression

$$
\left(x_{1}-\mathbf{i} y_{1}\right)\left(\alpha_{2}+\mathbf{i} \beta_{2}\right)-\left(x_{2}-\mathbf{i} y_{2}\right)\left(\alpha_{1}+\mathbf{i} \beta_{1}\right)
$$


may have nontrivial real part, but we can assume that it vanishes, because adding the cocycle such that $\alpha_{j}=L y_{j}$ and $\beta_{j}=-L x_{j}$ for some $L \in \mathbf{R}$ and $j=1,2$, it means changing the expression (5-2) by adding $2 L\left(x_{1} y_{2}-y_{1} x_{2}\right) \neq 0$.

Since (5-2) vanishes, there exist $\lambda \in \mathbf{R}$ and $\omega \in \mathbf{C}$ with $|\omega|=1$ such that

$$
\frac{\alpha_{2}+\mathbf{i} \beta_{2}}{x_{2}-\mathbf{i} y_{2}}=\frac{\alpha_{1}+\mathbf{i} \beta_{1}}{x_{1}-\mathbf{i} y_{1}}=\frac{\lambda}{2} \mathbf{i} \omega^{2} \text {. }
$$

Adding the coboundary such that $\alpha_{j}=\frac{\lambda}{2} y_{j}$ and $\beta_{j}=-\frac{\lambda}{2} x_{j}$, for $j=1,2$, we deduce:

$$
\begin{aligned}
& \alpha_{1}+\mathbf{i} \beta_{1}=\left(x_{1}-\mathbf{i} y_{1}\right) \frac{\lambda}{2} \mathbf{i} \omega^{2}+\frac{\lambda}{2}\left(y_{1}-\mathbf{i} x_{1}\right), \\
& \alpha_{2}+\mathbf{i} \beta_{2}=\left(x_{2}-\mathbf{i} y_{2}\right) \frac{\lambda}{2} \mathbf{i} \omega^{2}+\frac{\lambda}{2}\left(y_{2}-\mathbf{i} x_{2}\right) .
\end{aligned}
$$

Hence, expressing the scalar product · in terms of real parts, we have:

$$
\begin{aligned}
& \left(\alpha_{1}, \beta_{1}\right)=\alpha_{1}+\mathbf{i} \beta_{1}=\operatorname{Re}\left(\left(x_{1}-\mathbf{i} y_{1}\right) \mathbf{i} \omega\right) \lambda \omega=\left(\left(x_{1}, y_{1}\right) \cdot \mathbf{i} \omega\right) \lambda \omega, \\
& \left(\alpha_{2}, \beta_{2}\right)=\alpha_{2}+\mathbf{i} \beta_{2}=\operatorname{Re}\left(\left(x_{2}-\mathbf{i} y_{2}\right) \mathbf{i} \omega\right) \lambda \omega=\left(\left(x_{2}, y_{2}\right) \cdot \mathbf{i} \omega\right) \lambda \omega .
\end{aligned}
$$

And we conclude the proof of the lemma by linearity.

\section{Acknowledgments}

We thank Aris Daniilidis and Francesco Bonsante for useful conversations. The first author wishes to thank the Departament de Matemàtiques de la UAB for the kind hospitality during his frequent visits.

\section{References}

[Bart and Scannell 2007] A. Bart and K. P. Scannell, "A note on stamping", Geom. Dedicata 126 (2007), 283-291. MR 2008e:57014 Zbl 1130.57022

[Bestvina 1988] M. Bestvina, "Degenerations of the hyperbolic space”, Duke Math. J. 56:1 (1988), 143-161. MR 89m:57011 Zbl 0652.57009

[Boileau and Porti 2001] M. Boileau and J. Porti, "Geometrization of 3-orbifolds of cyclic type", Astérisque 272 (2001), 208. MR 2002f:57034 Zbl 0971.57004

[Boileau et al. 2005] M. Boileau, B. Leeb, and J. Porti, "Geometrization of 3-dimensional orbifolds", Ann. of Math. (2) 162:1 (2005), 195-290. MR 2007f:57028 Zbl 1087.57009

[Epstein and Penner 1988] D. B. A. Epstein and R. C. Penner, "Euclidean decompositions of noncompact hyperbolic manifolds", J. Differential Geom. 27:1 (1988), 67-80. MR 89a:57020 Zbl 0611.53036

[Johnson and Millson 1987] D. Johnson and J. J. Millson, "Deformation spaces associated to compact hyperbolic manifolds", pp. 48-106 in Discrete groups in geometry and analysis (New Haven, CT, 1984), edited by R. Howe, Progr. Math. 67, Birkhäuser, Boston, 1987. MR 88j:22010 Zbl 0664.53023

[Kapovich 1994] M. Kapovich, "Deformations of representations of discrete subgroups of SO(3, 1)", Math. Ann. 299:2 (1994), 341-354. MR 95d:57010 Zbl 0828.57009 
[Kapovich 2001] M. Kapovich, Hyperbolic manifolds and discrete groups, Progress in Mathematics 183, Birkhäuser, Boston, 2001. MR 2002m:57018 Zbl 0958.57001

[Kapovich 2007] M. Kapovich, “On sequences of finitely generated discrete groups”, preprint, 2007. arXiv 0708.2671

[Lubotzky and Magid 1985] A. Lubotzky and A. R. Magid, "Varieties of representations of finitely generated groups”, Mem. Amer. Math. Soc. 58:336 (1985), xi+117. MR 87c:20021 Zbl 0598.14042

[Morgan 1986] J. W. Morgan, "Group actions on trees and the compactification of the space of classes of SO(n, 1)-representations”, Topology 25:1 (1986), 1-33. MR 87h:20062 Zbl 0595.57030

[Morgan and Shalen 1984] J. W. Morgan and P. B. Shalen, "Valuations, trees, and degenerations of hyperbolic structures. I", Ann. of Math. (2) 120:3 (1984), 401-476. MR 86f:57011 Zbl 0583.57005

[Morgan and Shalen 1988] J. W. Morgan and P. B. Shalen, "Degenerations of hyperbolic structures. III. Actions of 3-manifold groups on trees and Thurston's compactness theorem", Ann. of Math. (2) 127:3 (1988), 457-519. MR 89e:57010b Zbl 0661.57004

[Paulin 1997] F. Paulin, "Sur les automorphismes extérieurs des groupes hyperboliques", Ann. Sci. École Norm. Sup. (4) 30:2 (1997), 147-167. MR 98c:20070 Zbl 0877.20014

[Scannell 2000] K. P. Scannell, "Infinitesimal deformations of some $\operatorname{SO}(3,1)$ lattices", Pacific J. Math. 194:2 (2000), 455-464. MR 2001c:57018 Zbl 1019.57007

[Scannell 2002] K. P. Scannell, "Local rigidity of hyperbolic 3-manifolds after Dehn surgery", Duke Math. J. 114:1 (2002), 1-14. MR 2003e:57031 Zbl 1025.57019

[Thurston 1980] W. P. Thurston, “The Geometry and Topology of Three-Manifolds", lecture notes, 1980, Available at http://www.msri.org/publications/books/gt3m/.

[Weil 1964] A. Weil, "Remarks on the cohomology of groups", Ann. of Math. (2) 80 (1964), 149157. MR 30 \#199 Zbl 0192.12802

[Wolf 1984] J. A. Wolf, Spaces of constant curvature, 5th ed., Publish or Perish, Houston, TX, 1984. MR 88k:53002 Zbl 0556.53033

Received August 1, 2008.

\author{
STEFANO FRANCAVIGLIA \\ Dipartimento di Matematica Applicata "U. Dini" \\ UNIVERSITÀ DEGLI STUDI DI PISA \\ VIA BUONARROTI 1C \\ 56127 PISA \\ ITALY \\ s.francaviglia@sns.it \\ http://www.dm.unipi.it/ francavi/
}

JOAN PORTI

DePaRtament DE Matemàtiques

UNIVERSITAT AUTÒNOMA DE BARCELONA

08193 BELLATERRA

SPAIN

porti@mat.uab.es

http://mat.uab.cat/ porti 